\title{
THE POTENTIAL OF GIS AS A MANAGEMENT TOOL FOR AVENUE TREES POPULATION IN SMALL COMMUNITIES; A CASE STUDY OF IDI-SHIN COMMUNITY, IBADAN, NIGERIA
}

\author{
O.S. Olokeogun ${ }^{\text {* }}$, O.O. Akintola ${ }^{\text {a }}$ E.K. Abodunrin ${ }^{\mathrm{a}}$ \\ ${ }^{\text {a }}$ Dept. of Forestry Technology, Federal College of Forestry, Nigeria - (Olokeogun, Akintola, Abodunrin) \\ forester-fisher@hotmail.com, toyinakintola73@gmail.com, ekolawole7@yahoo.com
}

Youth Forum

KEY WORDS: GIS, Avenue Trees, Urban Forest

\begin{abstract}
:
This study demonstrates the potentials of Geographic Information System (GIS) as a management tool for avenue trees (Street trees) populations in small communities (using Idi-Ishin community, Ibadan, Nigeria as a case study). GIS is a decision support system which integrate data or set of data from different sources, bringing them under the same referencing system in a computer system. An Ikonos Imagery (1m Spatial Resolution) of the study area was digitized to produce a digital map using ArcGIS 10.1 version. The avenue trees species $\geq 5 \mathrm{~cm}$ diameter at breast height $(\mathrm{DBH})$ was selected for enumeration. These trees were then measured and tagged. The Height, Girth and Geographic location (X \&Y coordinate) of the trees were measured with Haga altimeter, Girthing tape and Hand held Global Positioning System (GPS) respectively. The species and families of the trees enumerated were also identified. Data were analysed for basal area (BA) and volume (V). A total number of 43 avenue trees were assessed in Idi-Ishin Community. Roystonea regia accounted for the majority of the avenue trees (25.58\%), followed by Polyanthia longiflora (23.26\%), Gliricida seprium (20.93\%), Eucalyptus toreliana (13.95\%), Delunix regea (6.98\%). However Terminalia catapa, Terminalia radii, Azadrachita indica and Newbodia levis had the same abundance of $2.33 \%$. It was also observed that the benefits derived from these avenue trees includes; Carbon sequestration, Beautification, Wind break and shade. A spatial relational database was created for the assessed avenue trees using ArcCatalog of ArcGIS 10.1 version. Based on the findings from the study (which serves as baseline information for the management of the avenue trees in the study area), it was therefore recommended that subsequent assessment should be carried out at 3-5 year interval in other to ensure proper and continuous monitoring and updating of the data.
\end{abstract}

\section{INTRODUCTION}

Avenue (Street) tree is an important component of the urban forest. They are trees located on land forming or adjacent to a street, which have both direct and indirect effect on those using that street (Heimlich et al, 2008). With their canopies overhanging the roadway, street trees help to protect asphalt from deterioration by sunlight (Mcpherson et al, 1993) and reduce storm-water runoff from impervious surfaces (Nowak and Crane, 2002). Their shade keeps pedestrians, parked cars, and nearby buildings cool in the summer (Scott et al, 1999; Akbari, 2002). Avenue trees can also help to buffer the sights and sounds of nearby roadways (Fang and Ling, 2003) and reduce driving stress (Wolf, 2003). The appearance of commercial areas is also improved by the presence of avenue tree (Anderson and Cordell, 1988). Fuwape (2001), stated that, the benefits of street trees include: amelioration of weather pattern, provision of clean air, protection of biological diversity, protection of watershed, soil and food crops and provision of recreational facilities. Street trees can contribute significantly to human health. For instance, study has shown that children living in areas with more street trees had lower prevalence of asthma compared to those living where there are few street trees (Lovasi et al., 2008). Because of their proximity to pedestrians, cars, sidewalks and other infrastructure, street trees can be both major assets and major liabilities.

Despite the various benefits offered by street trees, little or no effort has been made to take full advantage of them. Therefore a need to encourage such arose and GIS is presumed to be a better and long lasting management tool. GIS is a decision support system which integrate data or set of data from different sources, bringing them under the same referencing system in a computer system. The Spatial analytical functions of GIS prove to aid better understanding of the street trees. GIS can aid planners, local elected officials, landscape architects, urban foresters, and non profit groups in quantifying benefits and costs of municipal trees (McPherson et al., 2002). GIS tend to offer Long-term management, reduction of tree program costs, and increasing street trees' ability to maintain benefits. The results of the various spatial analyses of GIS can be presented graphically and can also be used for broad regional studies or to create detailed small site assessments. The GIS model also contains algorithms for tree growth to allow users to create maps of future tree cover and estimate the benefits provided by those larger trees. This paper therefore examined the potentials of Geographic Information System (GIS) as a management tool for street tree (Avenue trees) populations in small communities, using Idi-shin Community as a case study.

\section{METHODOLOGY}

2.1

\section{THE STUDY AREA}

Idi-Isin Community is situated in Jericho, Ibadan North West Local Government Area of Oyo State, Nigeria. The area lies between longitude $3^{\circ} 51^{\prime} 33.19^{\prime \prime} \mathrm{E}$ to $3^{\circ} 51^{\prime} 3.56^{\prime \prime} \mathrm{E}$ and latitude $7^{\circ} 23^{\prime} 56.04 " \mathrm{~N}$ to $7^{\circ} 24^{\prime} 20.55^{\prime \prime} \mathrm{N}$. It has annual rainfall of about $1400 \mathrm{~mm}-1500 \mathrm{~mm}$ and average relative humidity of about $65 \%$. The average temperature is $32^{\circ} \mathrm{C}$ with two distinct seasons, which are dry, usually commences from November to 
March and rainy season. It is bounded by Jericho and Ile-titun community.

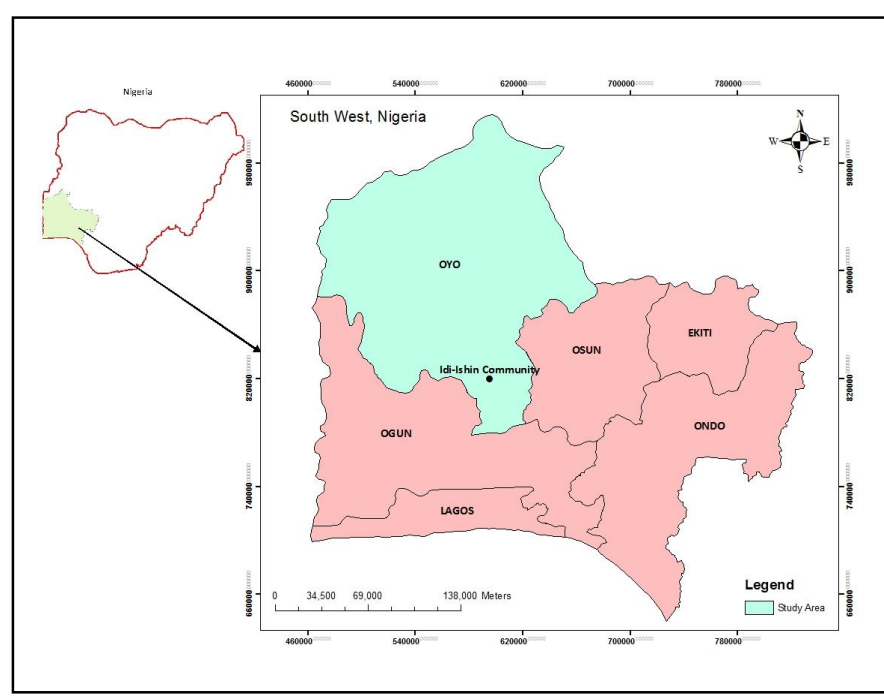

Figure 1. Location of the Study Area

\subsection{Data Source}

Both secondary and primary data source was used for this project. The secondary data collection includes;

i. An Ikonos Imagery (1m spatial resolution) of the study area, sourced from Google Earth

While the primary data include;

i. The tree species assessment data

ii. The coordinates of geographic features and location of the urban forest trees within the study area using Global Positioning System (GPS)

\subsection{Data Conversion}

The satellite image covering the study area was taken to the site for ground truthing. The imagery was then geo-referenced. Features (such as buildings and roads) were extracted (digitized) using ArcGIS 10.1 software. The roads were categorized into avenue, close, crescent, streets. The step by step approach (methodology) taken to achieve the stated objectives of this study are the following;

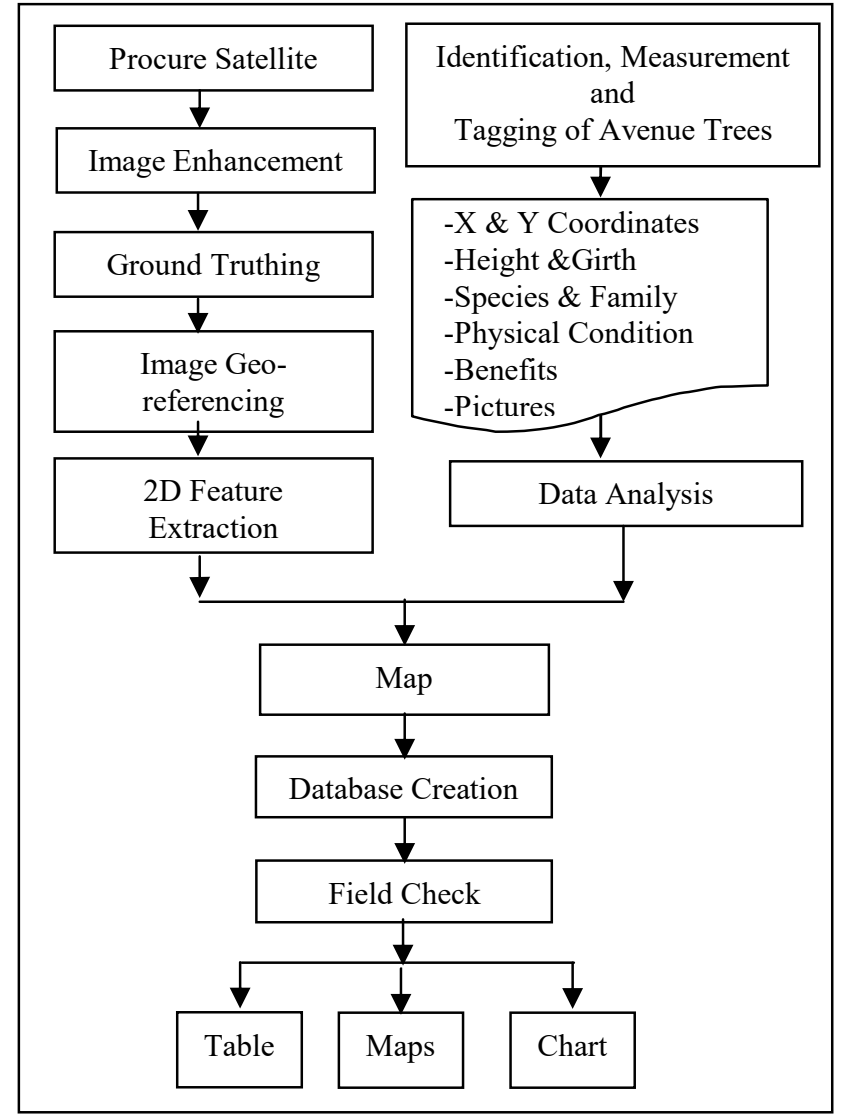

Figure 2. Flow Chart of the Research Methodology

\subsection{Database Creation}

A spatial database was created for the assessed avenue trees using ArcGIS 10.1 version. The attribute database was created by typing the attribute data into the themes attribute tables.

\section{SPATIAL ANALYSIS AND INFORMATION PRESENTATION}

\subsection{Map Production}

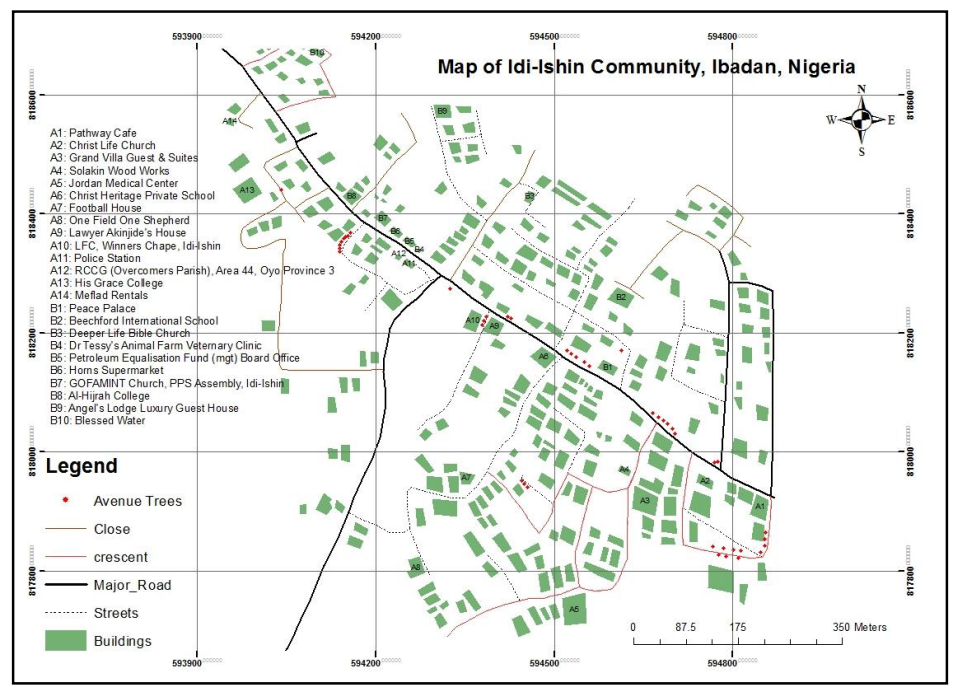

Figure 3. Produced Map of Idi-Ishin Community 


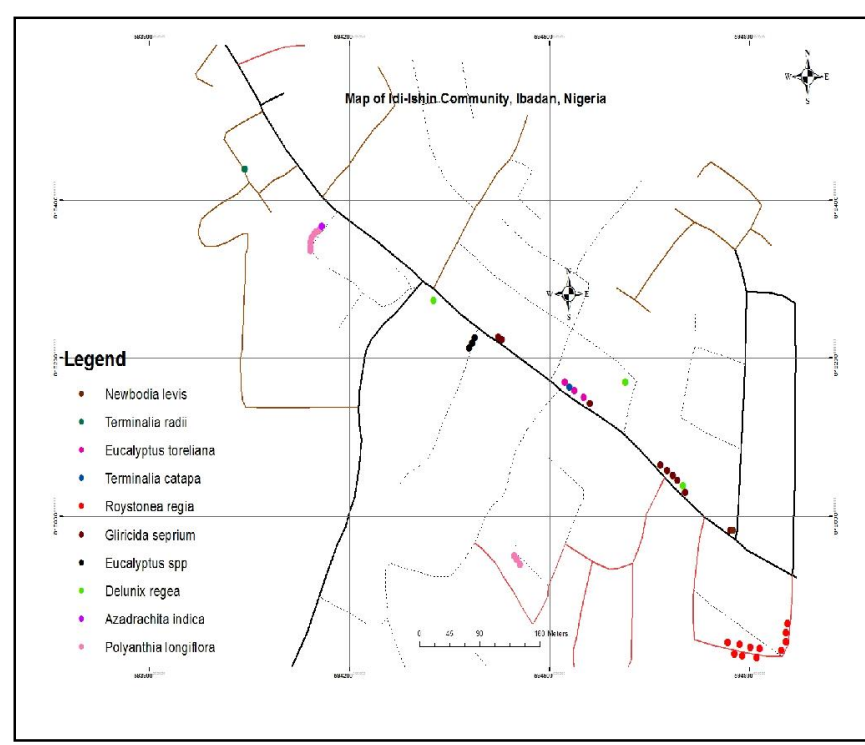

Figure 4. Spatial Distribution of Avenue Trees

3.2 Abundance and Composition

\begin{tabular}{|c|c|c|c|c|}
\hline $\mathbf{S} / \mathbf{N}$ & Name & $\begin{array}{lcc}X & \& & Y \\
\text { Coordinate } & \\
\end{array}$ & $\begin{array}{l}\text { DBH } \\
(\mathrm{cm})\end{array}$ & $\begin{array}{l}\text { Height } \\
(\mathrm{m})\end{array}$ \\
\hline 1 & $\begin{array}{l}\text { Roystonea } \\
\text { regia }\end{array}$ & $\begin{array}{lll}3^{0} & 51^{\prime} & 34.27^{\prime \prime} \mathrm{E} \\
7^{0} & 23^{\prime} & 56.06^{\prime \prime} \mathrm{N}\end{array}$ & 85 & 13.8 \\
\hline 2 & $\begin{array}{l}\text { Roystonea } \\
\text { regia }\end{array}$ & 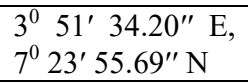 & 76 & 8.2 \\
\hline 3 & $\begin{array}{l}\text { Roystonea } \\
\text { regia }\end{array}$ & $\begin{array}{l}3^{0} 51^{\prime} 34.19^{\prime \prime} \mathrm{E} \\
7^{0} 23^{\prime} 55.30^{\prime \prime} \mathrm{N}\end{array}$ & 81 & 11.5 \\
\hline 4 & $\begin{array}{l}\text { Roystonea } \\
\text { regia }\end{array}$ & $\begin{array}{l}3^{0} 51^{\prime} 33.97^{\prime \prime} \mathrm{E} \\
7^{0} 23^{\prime} 54.95^{\prime \prime} \mathrm{N}\end{array}$ & 85 & 8.5 \\
\hline 5 & $\begin{array}{l}\text { Roystonea } \\
\text { regia }\end{array}$ & $\begin{array}{l}3^{0} 51^{\prime} 32.92^{\prime \prime} \text { E, } \\
7^{0} 23^{\prime} 55.04^{\prime \prime} \mathrm{N}\end{array}$ & 207 & 17.2 \\
\hline 6 & $\begin{array}{l}\text { Roystonea } \\
\text { regia }\end{array}$ & 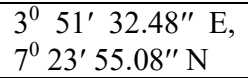 & 185 & 19.5 \\
\hline 7 & $\begin{array}{l}\text { Roystonea } \\
\text { regia }\end{array}$ & $\begin{array}{l}3^{0} 51^{\prime} 31.95^{\prime \prime} \mathrm{E} \\
7^{0} 23^{\prime} 55.20^{\prime \prime} \mathrm{N}\end{array}$ & 188 & 16.2 \\
\hline 8 & $\begin{array}{l}\text { Roystonea } \\
\text { regia }\end{array}$ & $\begin{array}{l}3^{0} 51^{\prime} 31.37^{\prime \prime} \mathrm{E} \\
7^{0} 23^{\prime} 55.28^{\prime \prime} \mathrm{N}\end{array}$ & 184 & 17 \\
\hline 9 & $\begin{array}{l}\text { Roystonea } \\
\text { regia }\end{array}$ & $\begin{array}{lll}3^{0} & 51^{\prime} & 32.77^{\prime \prime} \mathrm{E} \\
7^{0} & 23^{\prime} & 54.64^{\prime \prime} \mathrm{N}\end{array}$ & 170 & 16 \\
\hline 10 & $\begin{array}{l}\text { Roystonea } \\
\text { regia }\end{array}$ & $\begin{array}{ll}3^{0} & 51^{\prime} 32.07^{\prime \prime} \mathrm{E} \\
7^{0} & 23^{\prime} 54.72^{\prime \prime} \mathrm{N}\end{array}$ & 171 & 20 \\
\hline 11 & $\begin{array}{l}\text { Roystonea } \\
\text { regia }\end{array}$ & 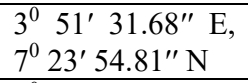 & 71 & 19.3 \\
\hline 12 & $\begin{array}{l}\text { Newbodia } \\
\text { levis }\end{array}$ & $\begin{array}{l}3^{0} 51^{\prime} 31.63^{\prime \prime} \mathrm{E} \\
7^{0} 23^{\prime} 59.88^{\prime \prime} \mathrm{N}\end{array}$ & 37 & 5.4 \\
\hline 13 & $\begin{array}{l}\text { Gliricida } \\
\text { seprium }\end{array}$ & $\begin{array}{l}3^{0} 51^{\prime} 31.44^{\prime \prime} \text { E, } \\
7^{0} 23^{\prime} 59.92^{\prime \prime} \mathrm{N}\end{array}$ & 35 & 5.6 \\
\hline 14 & $\begin{array}{l}\text { Gliricida } \\
\text { seprium }\end{array}$ & $\begin{array}{lll}3^{0} & 51^{\prime} & 29.25^{\prime \prime} \\
7^{0} & 24^{\prime} & 1.45^{\prime \prime} \mathrm{N}\end{array}$ & 43 & 5.7 \\
\hline 15 & Delunix regea & $\begin{array}{ll}3^{0} 51^{\prime} & 29.19^{\prime \prime} \text { E, } \\
7^{0} 24^{\prime} 1.71^{\prime \prime} \mathrm{N}\end{array}$ & 45 & 5.3 \\
\hline 16 & $\begin{array}{l}\text { Gliricida } \\
\text { seprium }\end{array}$ & $\begin{array}{lll}3^{0} & 51^{\prime} & 28.90^{\prime \prime} \\
7^{0} & 24^{\prime} & 1.97^{\prime \prime} \mathrm{N}\end{array}$ & 44 & 5.2 \\
\hline 17 & $\begin{array}{l}\text { Gliricida } \\
\text { seprium }\end{array}$ & $\begin{array}{ll}3^{0} & 51^{\prime} \quad 28.64^{\prime \prime} \text { E, } \\
7^{0} 24^{\prime} 2.17^{\prime \prime} \mathrm{N}\end{array}$ & 49 & 5.6 \\
\hline 18 & $\begin{array}{l}\text { Gliricida } \\
\text { seprium }\end{array}$ & $\begin{array}{lll}3^{0} & 51^{\prime} & 28.37^{\prime \prime} \text { E, } \\
7^{0} & 24^{\prime} & 2.27^{\prime \prime} \mathrm{N}\end{array}$ & 43 & 5.1 \\
\hline 19 & Gliricida & 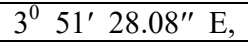 & 42 & 5.0 \\
\hline
\end{tabular}

\begin{tabular}{|c|c|c|c|c|}
\hline & seprium & $7^{0} 24^{\prime} 2.59^{\prime \prime} \mathrm{N}$ & & \\
\hline 20 & $\begin{array}{l}\text { Polyanthia } \\
\text { longiflora }\end{array}$ & $\begin{array}{lll}3^{0} & 51^{\prime} & 21.26^{\prime \prime} \text { E } \\
7^{0} & 23^{\prime} & 58.47^{\prime \prime} \mathrm{N}\end{array}$ & 33 & 7.2 \\
\hline 21 & $\begin{array}{l}\text { Polyanthia } \\
\text { longiflora }\end{array}$ & $\begin{array}{lll}3^{0} & 51^{\prime} & 21.19^{\prime \prime} \text { E } \\
7^{0} & 23^{\prime} & 58.57^{\prime \prime} \mathrm{N}\end{array}$ & 46 & 6.8 \\
\hline 22 & $\begin{array}{l}\text { Polyanthia } \\
\text { longiflora }\end{array}$ & $\begin{array}{l}3^{0} \quad 51^{\prime} \quad 20.90^{\prime \prime} \mathrm{E} \\
7^{0} \quad 23^{\prime} 58.96^{\prime \prime} \mathrm{N}\end{array}$ & 54 & 6.9 \\
\hline 23 & $\begin{array}{l}\text { Polyanthia } \\
\text { longiflora }\end{array}$ & $\begin{array}{lll}3^{0} & 51^{\prime} & 10.94^{\prime \prime} \text { E } \\
7^{0} & 24^{\prime} & 11.45^{\prime \prime} \mathrm{N}\end{array}$ & 55 & 8.5 \\
\hline 24 & $\begin{array}{l}\text { Polyanthia } \\
\text { longiflora }\end{array}$ & $\begin{array}{l}3^{0} 51^{\prime} 10.94^{\prime \prime} \mathrm{E} \\
7^{0} 24^{\prime} 11.68^{\prime \prime} \mathrm{N}\end{array}$ & 22 & 5.7 \\
\hline 25 & $\begin{array}{l}\text { Polyanthia } \\
\text { longiflora }\end{array}$ & $\begin{array}{lll}3^{0} & 51^{\prime} & 10.97^{\prime \prime} \mathrm{E} \\
7^{0} & 24^{\prime} & 11.84^{\prime \prime} \mathrm{N}\end{array}$ & 25 & 8.7 \\
\hline 26 & $\begin{array}{l}\text { Polyanthia } \\
\text { longiflora }\end{array}$ & $\begin{array}{l}3^{0} 51^{\prime} 11.01^{\prime \prime} \mathrm{E} \\
7^{0} 24^{\prime} 12.00^{\prime \prime} \mathrm{N}\end{array}$ & 27 & 7.0 \\
\hline 27 & $\begin{array}{l}\text { Polyanthia } \\
\text { longiflora }\end{array}$ & $\begin{array}{l}3^{0} 51^{\prime} 11.11^{\prime \prime} \mathrm{E} \\
7^{0} 24^{\prime} 12.17^{\prime \prime} \mathrm{N}\end{array}$ & 24 & 6.7 \\
\hline 28 & $\begin{array}{l}\text { Polyanthia } \\
\text { longiflora }\end{array}$ & $\begin{array}{l}3^{0} 51^{\prime} 11.27^{\prime \prime} \mathrm{E} \\
7^{0} 24^{\prime} 12.17^{\prime \prime} \mathrm{N}\end{array}$ & 28 & 7.6 \\
\hline 29 & $\begin{array}{l}\text { Polyanthia } \\
\text { longiflora }\end{array}$ & $\begin{array}{l}3^{0} 51^{\prime} 11.43^{\prime \prime} \mathrm{E} \\
7^{0} 24^{\prime} 12.36^{\prime \prime} \mathrm{N}\end{array}$ & 30 & 6.5 \\
\hline 30 & $\begin{array}{l}\text { Azadrachita } \\
\text { indica }\end{array}$ & $\begin{array}{lll}3^{0} & 51^{\prime} & 11.56^{\prime \prime} \mathrm{E} \\
7^{0} & 24^{\prime} & 12.49^{\prime \prime} \mathrm{N}\end{array}$ & 48 & 5.0 \\
\hline 31 & Delunix regea & $\begin{array}{l}3^{0} 51^{\prime} \quad 17.00^{\prime \prime} \mathrm{E} \\
7^{0} 24^{\prime} 9.39^{\prime \prime} \mathrm{N}\end{array}$ & 100 & 11.6 \\
\hline 32 & $\begin{array}{l}\text { Eucalyptus } \\
\text { spp }\end{array}$ & $\begin{array}{l}3^{0} 51^{\prime} 19.02^{\prime \prime} \mathrm{E} \\
7^{0} 24^{\prime} 7.89^{\prime \prime} \mathrm{N}\end{array}$ & 40 & 5.2 \\
\hline 33 & $\begin{array}{l}\text { Eucalyptus } \\
\text { spp }\end{array}$ & $\begin{array}{l}3^{0} 51^{\prime} 18.86^{\prime \prime} \text { E, } \\
7^{0} 24^{\prime} 7.66^{\prime \prime} \mathrm{N}\end{array}$ & 39 & 5.5 \\
\hline 34 & $\begin{array}{l}\text { Eucalyptus } \\
\text { spp }\end{array}$ & $\begin{array}{l}3^{0} 51^{\prime} 18.73^{\prime \prime} \mathrm{E} \\
7^{0} 24^{\prime} 7.43^{\prime \prime} \mathrm{N}\end{array}$ & 41 & 5.6 \\
\hline 35 & $\begin{array}{l}\text { Gliricida } \\
\text { seprium }\end{array}$ & $\begin{array}{lll}3^{0} & 51^{\prime} & 20.10^{\prime \prime} \\
7^{0} & 24^{\prime} & 7.88^{\prime \prime} \mathrm{N}\end{array}$ & 50 & 5.0 \\
\hline 36 & $\begin{array}{l}\text { Gliricida } \\
\text { seprium }\end{array}$ & $\begin{array}{l}3^{0} 51^{\prime} 20.33^{\prime \prime} \text { E, } \\
7^{0} 24^{\prime} 7.82^{\prime \prime} \mathrm{N}\end{array}$ & 48 & 5.6 \\
\hline 37 & $\begin{array}{l}\text { Eucalyptus } \\
\text { toreliana }\end{array}$ & $\begin{array}{lll}3^{0} & 51^{\prime} & 23.36^{\prime \prime} \\
7^{0} & 24^{\prime} & 6.09^{\prime \prime} \mathrm{N}\end{array}$ & 41 & 6.2 \\
\hline 38 & $\begin{array}{l}\text { Eucalyptus } \\
\text { toreliana }\end{array}$ & $\begin{array}{l}3^{0} 51^{\prime} 23.81^{\prime \prime} \mathrm{E} \\
7^{0} 24^{\prime} 5.66^{\prime \prime} \mathrm{N}\end{array}$ & 43 & 6.0 \\
\hline 39 & $\begin{array}{l}\text { Eucalyptus } \\
\text { toreliana }\end{array}$ & $\begin{array}{lll}3^{0} & 51^{\prime} & 24.34^{\prime \prime} \\
7^{0} & 24^{\prime} & 5.47^{\prime \prime} \mathrm{N}\end{array}$ & 40 & 5.9 \\
\hline 40 & $\begin{array}{l}\text { Terminalia } \\
\text { catapa }\end{array}$ & $\begin{array}{lll}3^{0} & 51^{\prime} & 23.59^{\prime \prime} \mathrm{E} \\
7^{0} & 24^{\prime} & 5.83^{\prime \prime} \mathrm{N}\end{array}$ & 43 & 6.0 \\
\hline 41 & $\begin{array}{l}\text { Gliricida } \\
\text { seprium }\end{array}$ & $\begin{array}{lll}3^{0} & 51^{\prime} & 24.60^{\prime \prime} \\
7^{0} & 24^{\prime} & 5.17^{\prime \prime} \mathrm{N}\end{array}$ & 44 & 5.6 \\
\hline 42 & Delunix regea & $\begin{array}{lll}3^{0} & 51^{\prime} & 26.42^{\prime \prime} \\
7^{0} & 24^{\prime} & 5.95^{\prime \prime} \mathrm{N}\end{array}$ & 49 & 7.4 \\
\hline 43 & $\begin{array}{l}\text { Terminalia } \\
\text { radii }\end{array}$ & $\begin{array}{l}3^{0} 51^{\prime} 7.72^{\prime \prime} \mathrm{E}, 7^{0} \\
24^{\prime} 14.91^{\prime \prime} \mathrm{N}\end{array}$ & 36 & 4.5 \\
\hline \multicolumn{3}{|c|}{ Mean } & 66.21 & 8.53 \\
\hline
\end{tabular}

Table 1. Trees Species Abundance with their Geo-Spatial Location

Table 1 presents the abundance and geo-spatial location of the tree species encountered. A total number of 43 avenue trees were assessed in Idi-Ishin Community. In terms of the species abundance, Roystonea regia accounted for the majority of the avenue trees $(25.58 \%)$, followed by Polyanthia longiflora (23.26\%), Gliricida seprium (20.93\%), Eucalyptus toreliana (13.95\%), Delunix regea (6.98\%). However Terminalia catapa, Terminalia radii, Azadrachita indica and Newbodia levis had the same abundance of $2.33 \%$. 
It was also observed from the study that the avenue trees are not well spatially distributed. The total Diameter at Breast Height (DBH) and Height of the assessed avenue trees species are 2847 $\mathrm{cm}$ and $366.8 \mathrm{~m}$ respectively.

3.3 Physical Conditions and Benefits of the Avenue Trees

\begin{tabular}{|c|c|c|c|}
\hline $\mathbf{S} / \mathbf{N}$ & NAME & $\begin{array}{l}\text { PHYSICAL } \\
\text { CONDITION }\end{array}$ & BENEFITS \\
\hline 1 & $\begin{array}{l}\text { Roystonea } \\
\text { regia }\end{array}$ & No physical defect & $\begin{array}{l}\text { Beautification, } \\
\text { Shade }\end{array}$ \\
\hline 2 & $\begin{array}{l}\text { Roystonea } \\
\text { regia }\end{array}$ & No physical defect & $\begin{array}{l}\text { Beautification, } \\
\text { Shade }\end{array}$ \\
\hline 3 & $\begin{array}{l}\text { Roystonea } \\
\text { regia }\end{array}$ & No physical defect & $\begin{array}{l}\text { Beautification, } \\
\text { Shade }\end{array}$ \\
\hline 4 & $\begin{array}{l}\text { Roystonea } \\
\text { regia }\end{array}$ & No physical defect & $\begin{array}{l}\text { Beautification, } \\
\text { Shade }\end{array}$ \\
\hline 5 & $\begin{array}{l}\text { Roystonea } \\
\text { regia }\end{array}$ & No physical defect & $\begin{array}{l}\text { Beautification, } \\
\text { Shade }\end{array}$ \\
\hline 6 & $\begin{array}{l}\text { Roystonea } \\
\text { regia }\end{array}$ & No physical defect & $\begin{array}{l}\text { Beautification, } \\
\text { Shade }\end{array}$ \\
\hline 7 & $\begin{array}{l}\text { Roystonea } \\
\text { regia }\end{array}$ & No physical defect & $\begin{array}{l}\text { Beautification, } \\
\text { Shade }\end{array}$ \\
\hline 8 & $\begin{array}{l}\text { Roystonea } \\
\text { regia }\end{array}$ & No physical defect & $\begin{array}{l}\text { Beautification, } \\
\text { Shade }\end{array}$ \\
\hline 9 & $\begin{array}{l}\text { Roystonea } \\
\text { regia }\end{array}$ & No physical defect & $\begin{array}{l}\text { Beautification, } \\
\text { Shade }\end{array}$ \\
\hline 10 & $\begin{array}{l}\text { Roystonea } \\
\text { regia }\end{array}$ & No physical defect & $\begin{array}{l}\text { Beautification, } \\
\text { Shade }\end{array}$ \\
\hline 11 & $\begin{array}{l}\text { Roystonea } \\
\text { regia }\end{array}$ & No physical defect & $\begin{array}{l}\text { Beautification, } \\
\text { Shade }\end{array}$ \\
\hline 12 & $\begin{array}{l}\text { Newbodia } \\
\text { levis }\end{array}$ & No physical defect & $\begin{array}{l}\text { Carbon } \\
\text { sequestration, } \\
\text { Shade }\end{array}$ \\
\hline 13 & $\begin{array}{l}\text { Gliricida } \\
\text { seprium }\end{array}$ & No physical defect & $\begin{array}{l}\text { Carbon } \\
\text { sequestration, } \\
\text { Shade } \\
\end{array}$ \\
\hline 14 & $\begin{array}{l}\text { Gliricida } \\
\text { seprium }\end{array}$ & $\begin{array}{ll}\text { No } & \text { physical } \\
\text { defect, } & \text { requires } \\
\text { pruning } & \\
\end{array}$ & $\begin{array}{l}\text { Carbon } \\
\text { sequestration, } \\
\text { Shade }\end{array}$ \\
\hline 15 & Delunix regea & $\begin{array}{ll}\text { No } & \text { physical } \\
\text { defect, } & \text { requires } \\
\text { pruning } & \\
\end{array}$ & $\begin{array}{l}\text { Carbon } \\
\text { sequestration, } \\
\text { Shade } \\
\end{array}$ \\
\hline 16 & $\begin{array}{l}\text { Gliricida } \\
\text { seprium }\end{array}$ & $\begin{array}{ll}\text { No } & \text { physical } \\
\text { defect, } & \text { requires } \\
\text { pruning } & \\
\end{array}$ & $\begin{array}{l}\text { Carbon } \\
\text { sequestration, } \\
\text { Shade } \\
\end{array}$ \\
\hline 17 & $\begin{array}{l}\text { Gliricida } \\
\text { seprium }\end{array}$ & $\begin{array}{ll}\text { No } & \text { physical } \\
\text { defect, } & \text { requires } \\
\text { pruning } & \\
\end{array}$ & $\begin{array}{l}\text { Carbon } \\
\text { sequestration, } \\
\text { Shade }\end{array}$ \\
\hline 18 & $\begin{array}{l}\text { Gliricida } \\
\text { seprium }\end{array}$ & $\begin{array}{ll}\text { No } & \text { physical } \\
\text { defect, } & \text { requires } \\
\text { pruning } & \\
\end{array}$ & $\begin{array}{l}\text { Carbon } \\
\text { sequestration, } \\
\text { Shade } \\
\end{array}$ \\
\hline 19 & $\begin{array}{l}\text { Gliricida } \\
\text { seprium }\end{array}$ & $\begin{array}{ll}\text { No } & \text { physical } \\
\text { defect, } & \text { requires } \\
\text { pruning } & \\
\end{array}$ & $\begin{array}{l}\text { Carbon } \\
\text { sequestration, } \\
\text { Shade } \\
\end{array}$ \\
\hline 20 & $\begin{array}{l}\text { Polyanthia } \\
\text { longiflora }\end{array}$ & No physical defect & $\begin{array}{l}\text { Carbon } \\
\text { sequestration, } \\
\text { Beautification, } \\
\text { Wind break } \\
\end{array}$ \\
\hline 21 & $\begin{array}{l}\text { Polyanthia } \\
\text { longiflora }\end{array}$ & No physical defect & $\begin{array}{l}\text { Carbon } \\
\text { sequestration, } \\
\text { Beautification, } \\
\text { Wind break }\end{array}$ \\
\hline
\end{tabular}

\begin{tabular}{|c|c|c|c|}
\hline 22 & $\begin{array}{l}\text { Polyanthia } \\
\text { longiflora }\end{array}$ & No physical defect & $\begin{array}{l}\text { Carbon } \\
\text { sequestration, } \\
\text { Beautification, } \\
\text { Wind break }\end{array}$ \\
\hline 23 & $\begin{array}{l}\text { Polyanthia } \\
\text { longiflora }\end{array}$ & No physical defect & $\begin{array}{l}\text { Carbon } \\
\text { sequestration, } \\
\text { Beautification, } \\
\text { Wind break }\end{array}$ \\
\hline 24 & $\begin{array}{l}\text { Polyanthia } \\
\text { longiflora }\end{array}$ & No physical defect & $\begin{array}{l}\text { Carbon } \\
\text { sequestration, } \\
\text { Beautification, } \\
\text { Wind break } \\
\end{array}$ \\
\hline 25 & $\begin{array}{l}\text { Polyanthia } \\
\text { longiflora }\end{array}$ & No physical defect & $\begin{array}{l}\text { Carbon } \\
\text { sequestration, } \\
\text { Beautification, } \\
\text { Wind break }\end{array}$ \\
\hline 26 & $\begin{array}{l}\text { Polyanthia } \\
\text { longiflora }\end{array}$ & No physical defect & $\begin{array}{l}\text { Carbon } \\
\text { sequestration, } \\
\text { Beautification, } \\
\text { Wind break }\end{array}$ \\
\hline 27 & $\begin{array}{l}\text { Polyanthia } \\
\text { longiflora }\end{array}$ & No physical defect & $\begin{array}{l}\text { Carbon } \\
\text { sequestration, } \\
\text { Beautification, } \\
\text { Wind break }\end{array}$ \\
\hline 28 & $\begin{array}{l}\text { Polyanthia } \\
\text { longiflora }\end{array}$ & No physical defect & $\begin{array}{l}\text { Carbon } \\
\text { sequestration, } \\
\text { Beautification, } \\
\text { Wind break } \\
\end{array}$ \\
\hline 29 & $\begin{array}{l}\text { Polyanthia } \\
\text { longiflora }\end{array}$ & No physical defect & $\begin{array}{l}\text { Carbon } \\
\text { sequestration, } \\
\text { Beautification, } \\
\text { Wind break }\end{array}$ \\
\hline 30 & $\begin{array}{l}\text { Azadrachita } \\
\text { indica }\end{array}$ & No physical defect & $\begin{array}{l}\text { Carbon } \\
\text { sequestration, } \\
\text { Beautification, } \\
\text { Wind break } \\
\end{array}$ \\
\hline 31 & Delunix regea & No physical defect & $\begin{array}{l}\text { Carbon } \\
\text { sequestration, } \\
\text { Shade } \\
\end{array}$ \\
\hline 32 & $\begin{array}{l}\text { Eucalyptus } \\
\text { spp }\end{array}$ & No physical defect & $\begin{array}{l}\text { Carbon } \\
\text { sequestration }\end{array}$ \\
\hline 33 & $\begin{array}{l}\text { Eucalyptus } \\
\text { spp }\end{array}$ & No physical defect & $\begin{array}{l}\text { Carbon } \\
\text { sequestration }\end{array}$ \\
\hline 34 & $\begin{array}{l}\text { Eucalyptus } \\
\text { spp }\end{array}$ & No physical defect & $\begin{array}{l}\text { Carbon } \\
\text { sequestration }\end{array}$ \\
\hline 35 & $\begin{array}{l}\text { Gliricida } \\
\text { seprium }\end{array}$ & $\begin{array}{ll}\text { No } & \text { physical } \\
\text { defect, } & \text { requires } \\
\text { pruning } & \\
\end{array}$ & $\begin{array}{l}\text { Carbon } \\
\text { sequestration }\end{array}$ \\
\hline 36 & $\begin{array}{l}\text { Gliricida } \\
\text { seprium }\end{array}$ & $\begin{array}{ll}\text { No } & \text { physical } \\
\text { defect, } & \text { requires } \\
\text { pruning } & \\
\end{array}$ & $\begin{array}{l}\text { Carbon } \\
\text { sequestration }\end{array}$ \\
\hline 37 & $\begin{array}{l}\text { Eucalyptus } \\
\text { toreliana }\end{array}$ & No physical defect & $\begin{array}{l}\text { Carbon } \\
\text { sequestration, } \\
\text { Shade }\end{array}$ \\
\hline 38 & $\begin{array}{l}\text { Eucalyptus } \\
\text { toreliana }\end{array}$ & No physical defect & $\begin{array}{l}\text { Carbon } \\
\text { sequestration, } \\
\text { Shade } \\
\end{array}$ \\
\hline 39 & $\begin{array}{l}\text { Eucalyptus } \\
\text { toreliana }\end{array}$ & No physical defect & $\begin{array}{l}\text { Carbon } \\
\text { sequestration, } \\
\text { Shade }\end{array}$ \\
\hline 40 & $\begin{array}{l}\text { Terminalia } \\
\text { catapa }\end{array}$ & No physical defect & $\begin{array}{l}\text { Carbon } \\
\text { sequestration, } \\
\text { Shade } \\
\end{array}$ \\
\hline 41 & $\begin{array}{l}\text { Gliricida } \\
\text { seprium }\end{array}$ & No physical defect & $\begin{array}{l}\text { Carbon } \\
\text { sequestration, }\end{array}$ \\
\hline
\end{tabular}




\begin{tabular}{|l|l|l|l|}
\hline 42 & Delunix regea & No physical defect & $\begin{array}{l}\text { Shade } \\
\text { sequestration }\end{array}$ \\
\hline 43 & $\begin{array}{l}\text { Terminalia } \\
\text { radii }\end{array}$ & No physical defect & $\begin{array}{l}\text { Carbon } \\
\text { sequestration, } \\
\text { Beautification }\end{array}$ \\
\hline
\end{tabular}

Table 2. Idi-Ishin Avenue Trees Physical Conditions and Benefits

Table 2 presents the physical conditions of individual trees species and benefits from them. Physical observations from the avenue trees revealed that majority of trees had no physical defect, while some of the trees needed to be pruned. According to Britt (2008), poor pruning causes decay that may lead to severe damages to healthy trees.

It was also observed that the benefits derived from these avenue trees includes; Carbon sequestration, Beautification, Wind break and shade, which is in accordance with Fuwape (2001), who stated that, the benefits of street trees include: amelioration of weather pattern, provision of clean air, protection of biological diversity, protection of watershed, soil and food crops and provision of recreational facilities

\subsection{Database Query}

Decision making requires information generated from spatial search and necessary operations performed. The database and graphics were manipulated and processed to generate information about the entities (spatial features) for GIS analysis. Data were retrieved from the database i.e. graphics and tabular data using ArcGIS 10.1. GIS analysis helps to ascertain the fitness of the database.

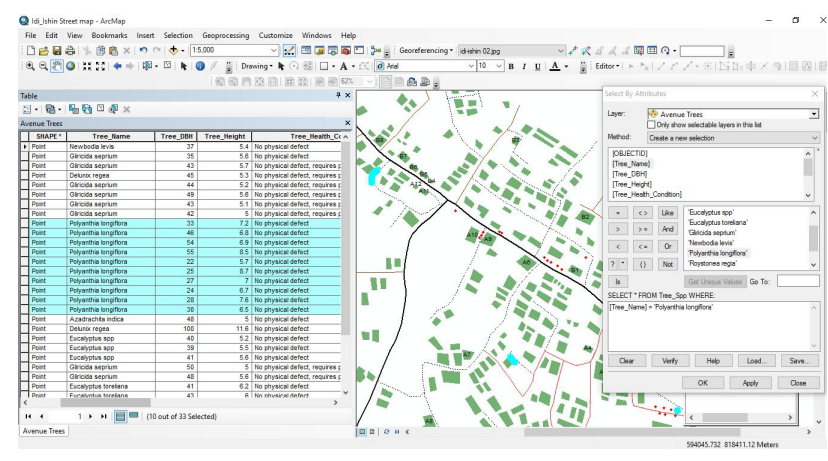

Figure 4. Query for Avenue trees (Polyanthia longiflora) available in the study area

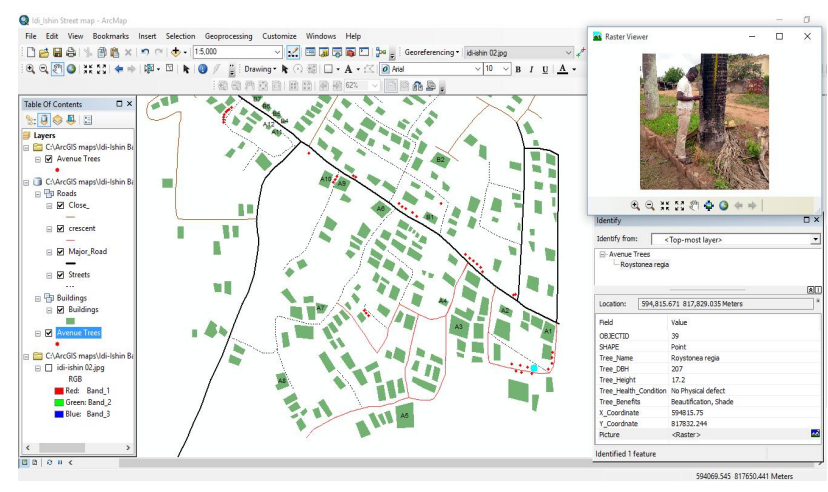

Figure 5. Query for Individual Avenue Tree

\section{CONCLUSION AND RECOMMENDATION}

\subsection{Conclusion}

Sustainable management of avenue trees is highly essential because of their environmental benefits such as energy conservation, carbon storage, air pollution abatement, storm water runoff reduction, etc. They are also known to enhance real estate value, tourism and commerce and citizen quality of life. With all these benefits that avenue trees offers; its calls for proper management. GIS as a decision support system has proven to be an effective and efficient tool for the management of these avenue trees populations in small communities.

\subsection{Recommendation}

The findings from this study serve as baseline information for the management of the avenue trees population in the study area. Subsequent assessment of the avenue trees at 3-5 year interval is recommended in other to ensure proper and continuous monitoring and updating of the data. The use of GIS as a tool to manage avenue trees is also recommended to Local Government Authorities, State Government, Forestry Research Institute of Nigeria (FRIN), Forest Managers, etc, as GIS spatial analysis ensures efficient and economical ways for handling spatial problems. GIS will also ensure proper and continuous monitoring and updating of information about urban forest.

\section{REFERENCES}

Akbari, H., 2002. Shade trees reduce building energy use and $\mathrm{CO}_{2}$ emissions from power plants. Environmental Pollution 116:S119-S126.

Anderson, L.M., and Cordell, K.K., 1988. Influence of trees on residential property values in Athens, Georgia (U.S.A): A survey based on actual sale prices. Landscape and Urban Planning 15:153-164.

Britt, C., 2008. Trees in Town II ; A new Survey of Urban Trees in England and Their Condition and Management Department for Communities and Local Government, $646 \mathrm{pp}$.

Fang, C., And Ling, D., 2003. Investigation of the noise reduction provided by tree belts Landscape and Urban Planning 63(4):187-195

Fuwepa, J.A., 2001. Forest Resources and Economic Development in Ondo State. Paper presented at Ondo State Economic Summit. 1-15pp.

Heimlich, J.T.D. Sydnor, Bumgardner M., and O'Brien P., 2008. Attitudes of Residents Towards Street Trees on Four Species in Toledo, Ohio, U.S. Before Removal of Ash Trees (Fraxinus spp) from Emerald Ash Borer (Agrilus planipennis) Arboriculture \& Urban Forestry 34(1):47-53

Nowak, D.J., Crane, D.E., and Stevens, J.C., 2006. Air pollution removal by urban trees and shrubs in the United States. Urban Forestry \& Urban Greening 4:115123

McPherson, E.G., and Rowntree, R.A., 1993. Energy Conservation Potentials of Urban Tree Planting. Journal of Aboriculture 19(6):321-331

Lovasi, G.S., Quinn, J.W., Neckerman, K.M., Perzanowski, M.S., and Rundle, A., 2008. Children Living in Areas with More Street Trees Have Lover Prevalence of 
The International Archives of the Photogrammetry, Remote Sensing and Spatial Information Sciences, Volume XLI-B6, 2016 XXIII ISPRS Congress, 12-19 July 2016, Prague, Czech Republic

Asthma. Journal of Epidemiology and Community Health, 62, 647-649

Scott, K.I., Simpson, J.R., and McPherson, E.G., 1999. Effects of tree cover on parking lot microclimate and vehicle emissions. Journal of Aboriculture 25(3): 129-142

Wolf, K.L., 2003. Freeway roadside management: The urban forest beyond the while line. Journal of Arboriculture 29(3):127-136 